
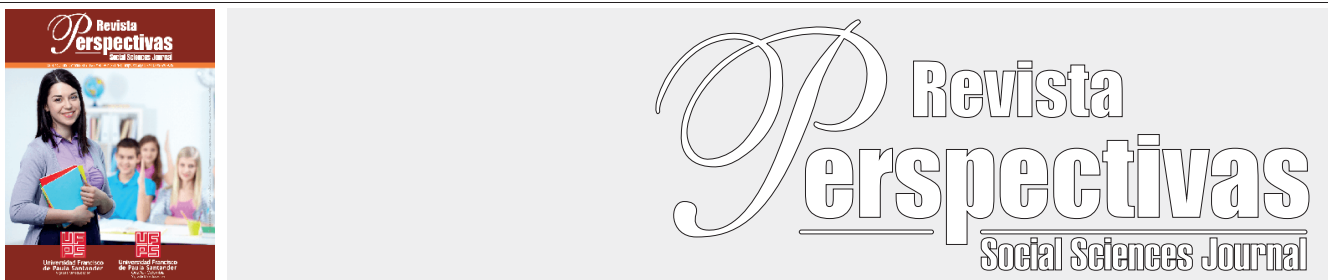

Artículo Original

https://doi.org/10.22463/0122820X.1793

\title{
Gobernanza y participación para el desarrollo de estudiantes como sujetos políticos
}

Governance and participation for the development of students as political subjects

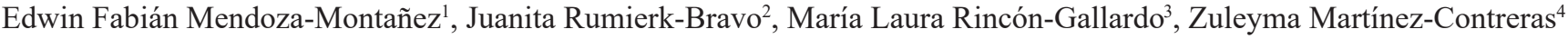

${ }^{\prime}$ Magister en Gestión de la Tecnología Educativa, ORCID 0000-0003-2740-0090, edwinfabianmm@ufps.edu.co, Colegio San Francisco de Sales, Cúcuta, Colombia.

${ }^{2}$ Trabajadora Social, juanitarumierkb@gmail.com, ORCID 0000-0001-9640-3653, Universidad Simón Bolivar, Cúcuta, Colombia.

${ }^{3}$ Trabajadora Social (en formación), marialaurarg@ufps.edu.co, ORCID 0000-0002-7316-3930, Universidad Francisco de Paula Santander Cúcuta, Colombia.

${ }^{4}$ Trabajadora Social (en formación), zuleymamc@ufps.edu.co, ORCID 0000-0002-6641-4447, Universidad Francisco de Paula Santander Cúcuta, Colombia.

Cómo citar: E. Mendoza, J. Rumierk, M. Rincón y Z. Martínez, "Gobernanza y participación para el desarrollo de estudiantes como sujetos políticos”. Perspectivas, vol. 4, no. 2, pp. 39-46, 2019.

Recibido: Junio 27, 2018; Aprobado: Octubre 03, 2018.

\begin{tabular}{ll}
\hline RESUMEN \\
\hline Palabras clave: & $\begin{array}{l}\text { El presente texto se abordará en torno al ejercicio de la gobernanza y su uso como herramienta para el desarrollo } \\
\text { de estudiantes como sujetos políticos, en donde se analizará de qué manera la participación contribuye al }\end{array}$ \\
desarrollo de la gobernanza en los estudiantes, tomando parte de los diferentes postulados teóricos que \\
Participación & permiten su abordaje, avanzando en la comprensión de esta en el contexto educativo colombiano, reconociendo \\
Política & la importancia de la escuela como formadora de competencias ciudadanas en los estudiantes y su participación \\
Sujetos políticos & política; en efecto, se realizó por medio del enfoque cualitativo, con nivel explicativo dado a que se busca \\
Educación & responder a la causa de un fenómeno social empleando el método fenomenológico, pues este permite analizar \\
& el sujeto de estudio a partir de la realidad que este vive, tanto interna como externamente, pues dicha realidad \\
& radica su esencia en el modo como el sujeto vive y percibe el fenómeno; finalmente se comprenderá que el \\
& modelo de la gobernanza resulta importante para la formación política participativa en estudiantes desde la \\
& escuela, siendo está el primer contexto educativo en donde se forman las ideologías y subjetividades que \\
regirán a las personas a lo largo de su vida.
\end{tabular}

\begin{tabular}{|c|c|}
\hline & ABSTRACT \\
\hline Keywords: & The present text will be approached around the exercise of governance and its use as a tool for the development \\
\hline Governance & $\begin{array}{l}\text { of students as political subjects, where it will be analyzed how participation contributes to the development } \\
\text { of governance in students, taking part in the different theoretical postulates that allow its approach, advancing }\end{array}$ \\
\hline Governance & of governance in students, taking part in the different theoretical postulates that allow its approach, advancing \\
\hline Participation & it in the Colombian educational context, recognizing the importance of the school \\
\hline Policy & mpetencies in students and their political participation; In effect, it was carried out \\
\hline Political subjects & $\begin{array}{l}\text { through a qualitative approach, with an explanatory level given that it seeks to respond to the cause of a social } \\
\text { phenomenon by using the phenomenological method, since this allows for the analysis of the subject of study }\end{array}$ \\
\hline Education & $\begin{array}{l}\text { based on the reality that he or she lives, both internally and externally, since this reality lies in the way that } \\
\text { the subject lives and perceives the phenomenon; Finally, it will be understood that the model of governance is } \\
\text { important for participatory political education in students from school, being the first educational context where } \\
\text { the ideologies and subjectivities that will govern people throughout their lives are formed. }\end{array}$ \\
\hline
\end{tabular}

\section{Introducción}

La historia de Colombia a lo largo del tiempo ha estado enmarcada en la ausencia de la intervención de la sociedad como ciudadanía en el ámbito político y gubernamental de la nación, en efecto, esto produce que las decisiones del Estado se desarrollen bajo inequidad e injusticia social, lo cual afecta en gran manera a los pobladores colombianos; la participación, posee real influencia durante la toma de decisiones del Estado, no solo en temas tangenciales, sino en la construcción de caminos que sean seguros para la superación de la opresión e injustica, caminos que sean orientados hacia relaciones equitativas, simétricas y horizontales (Insuasty, 2008). Lo cierto es que en los últimos años, debido a los múltiples escándalos de corrupción ha producido que las personas cada 
vez más se alejen de su papel como sujetos políticos, como agentes de cambio y como actores sociales, rol que es fundamental como ciudadano para el desarrollo de la nación, dado que es necesario "Una sociedad que no comulgue con la corrupción, que denuncie, que no le tema a la represión y que promueva una cultura del bienestar general por encima de los intereses particulares" (Parada, 2010).

El ser humano por naturaleza posee posturas políticas, es decir, ser sujeto político es inherente a su naturaleza, pero la complejidad radica en que dicha naturaleza o rol, sea desarrollada, puesto que:

Como seres humanos poseemos unas características comunes: somos seres sociales, culturales y políticos que, permeados por el contexto y entorno de formación, apropiamos ciertas formas y maneras de asumir y leer el mundo. Desde los diferentes escenarios que allí emergen se va estableciendo una construcción de sujeto y subjetividades en un marco social legítimamente propio. (Peña, 2017, p.3)

Siendo necesario entender que un sujeto político es formado al momento de enfrentar una restructuración de una subjetividad colectiva, que va en busca del cambio de la supremacía de un estado sobre otros, permitiendo evidenciar proceso de identificación, pero sobre todo de autonomía es por esto que se considera que los:

Sujetos políticos y su potencialidad para invocar el momento fundacional de lo político pueden ser complementadas tanto con el análisis de los procesos de identidad como de aquellos que conciernen a la lucha hegemónica por la ordenación social, la cual incluye la emergencia de sujetos políticos democráticos que disputan aspectos puntuales del orden como sujetos políticos populares producto de articulaciones capaces de emprender acciones (Retamozo, 2009, p. 86).

En consecuencia, a lo anterior, la gobernanza surge como herramienta para una nueva construcción de gobierno, en palabras de Le Gales (1998) "un proceso de coordinación de actores, de grupos sociales, de instituciones para lograr metas definidas colectivamente en entornos fragmentados" (p.7). Es decir, es una construcción, no en manera vertical, sino de forma horizontal que surja desde las comunidades, los actores sociales, los ciudadanos; en donde se articule el gobierno y los ciudadanos para la edificación de la nación, es decir la gobernanza, término relativamente nuevo para América Latina, propone la participación de los ciudadanos en el desarrollo de políticas públicas, estatales y decisiones gubernamentales para el desarrollo de la nación.

La noción de gobernanza pone énfasis en el carácter policéntrico de los sistemas políticos y de los procesos de toma de decisiones públicas. Parte de reconocer la diversidad de actores en los escenarios públicos y del papel que pueden jugar en la construcción de futuros colectivos. (Ruano, 2002, p.6).

En consecuencia, a lo anterior, vale la pena resaltar que en la generación promotora del cambio y la reestructuración de una sociedad se encuentra los jóvenes, es por ello de suma importancia promover desde la escuela la participación y desarrollo de sujetos políticos, dado que:

Desde la escuela los niños experimentan relaciones de convivencia y participación tangibles, las cuales van interiorizando en su actuar y son adaptadas a su ethos cotidiano como costumbre o forma de vida, esto es innegable. La escuela entonces se erige como un espacio donde se fragua la cultura de la participación, del respeto y del bienestar común. (Parada, 2010, p.650)

La escuela es entonces, un espacio académico que contribuye a la construcción de subjetividades, facilitador de la creación de una cultura que promueva la participación, para de este modo generar cambios y transformaciones, en otras palabras

La construcción de subjetividades es un tema que se abre paso dentro de la dinámica académica, educativa y escolar. Pensar en la configuración del sujeto es la inquietud, preocupación y tema de investigación que genera diversos interrogantes que abren las puertas a múltiples formas de análisis en escenarios como la educación. (Peña 2017, p. 229).

Ratificando que el aula de clase es el lugar donde se fomentan la construcción de una persona como 
participante político y social en su investigación "Los niños y las niñas, ¿sujetos políticos?: construcciones posibles desde la escuela y el aula"

Con todo lo anterior se sigue ratificando que la escuela, toma un papel fundamental en la formación de personas como sujetos políticos, dado que allí es uno de los escenarios principales en donde los estudiantes piensan, reflexionan y adquieren saberes bases para el desarrollo de su vida, es en este momento en donde surgen interrogantes, cuestionamientos, que abordados de manera correcta permitirán el crecimiento de su pensamiento, desarrollaran posturas e ideologías que posiblemente los acompañaran por el resto de su vida. Llevando a enfatizar la postura que "la educación de las masas es el problema fundamental de los países en desarrollo, una educación que, liberada de todos los rasgos alienantes, constituya una fuerza posibilitadora del cambio y sea impulso de libertad" (Freire, 1997)

En el transcurso de los últimos años, se ha evidenciado en el escenario de participación política de Colombia, la visibilización de los jóvenes y su deseo de cambio y transformación de la nación, según el Departamento Nacional de Estadística, la población joven de Colombia, (14-28 años) se encuentra en 12.757.040 personas: 6.242.436 mujeres y 6.514.604 hombres (DANE, 2017), sin embargo constitucionalmente, la edad para el sufragio son los 18 años, la cifra de la población joven es considerablemente importante, pues si se logra la participación política de los jóvenes y su formación como sujetos políticos desde temprana edad, se lograran resultados significativos en un futuro, es por ello que la población elegida para desarrollar la presente investigación son jóvenes entre los 14 y 16 años.

Por otra parte, el estudio de la gobernanza y la participación para el desarrollo de sujetos políticos es un tema que aborda holísticamente a la comunidad y la sociedad en general, dado que, es función de los ciudadanos de un país, ser partícipes de las decisiones gubernamentales que se toman en su nación, ejerciendo así su rol como sujetos políticos, pues lo que sucede en torno a ellos, no es una realidad ajena, desde esta instancia, se halla la importancia de la presente investigación, pues si bien es cierta la relevancia de desarrollar en cada ciudadano su papel como sujeto político, el escenario inicial para desempeñarlo y formarlo como tal, sería entonces "La escuela ya que ha sido el espacio de socialización desde temprana edad y donde se tejen las primeras relaciones jerárquicas, después del hogar" (Monsalve, 2016).

Según a lo anterior cabe resaltar que el trabajo social es definido según el Código de Ética Profesional de los Trabajadores Sociales en Colombia como:

la profesión ubicada en el campo de las ciencias sociales y humanas, que interviene en procesos y proyectos relacionados con las políticas de bienestar y desarrollo humano y social, teniendo como fundamento los meta criterios de la democracia: bien común, justicia y libertad, en el marco de la actividad profesional. (Consejo Nacional de Trabajo Social, 2013, p. 23)

En consecuencia, a lo anterior y desde la mirada disciplinar del trabajo social y su rol en el empoderamiento de las personas, surge la presente investigación, titulada, Gobernanza y participación para el desarrollo de sujetos políticos.

\section{Materiales y métodos}

El enfoque bajo el cual se desarrollará la presente investigación, es el cualitativo, en consecuencia, al sentido epistemológico de las Ciencias Sociales y el Trabajo Social; la investigación cualitativa es definida como aquella que:

Estudia la realidad en su contexto natural, tal y como sucede, intentando sacar sentido de, o interpretar los fenómenos de acuerdo con los significados que tienen para las personas implicadas. La investigación cualitativa implica la utilización y recogida de una gran variedad de materialesentrevista, experiencia personal, historias de vida, observaciones, textos históricos, imágenes, sonidos - que describen la rutina y las situaciones problemáticas y los significados en la vida de las personas. (Rodríguez, Gil y Garcia,1996, p.32)

Es decir, la investigación cualitativa es aquella que estudia el objeto de estudio en su contexto 
natural, para ello el investigador genera un grado de acercamiento con el fenómeno lo cual le permite adentrarse en la investigación, en donde cada persona que participa como objeto de investigación es portadora de diversos significados, añadido a ello, la investigación cualitativa va más allá de la corriente positivista, pues permite analizar aspectos a mayor profundidad por medio del contacto directo que le permiten comprender la complejidad de la realidad humana.

En consecuencia, a lo anterior, la presente investigación es de tipo explicativa dado a que pretender responder a la causa de un fenómeno social, cabe resaltar que los estudios explicativos son aquellos que:

Van más allá de la descripción de conceptos o fenómenos o del establecimiento de relaciones entre conceptos; están dirigidos a responder a las causas de los eventos físicos o sociales. Como su nombre lo indica, su interés se centra en explicar por qué ocurre un fenómeno y en qué condiciones se da éste, o por qué dos o más variables están relacionadas. (Hernández, Fernández y Baptista, 1997, p.18)

A parte de ello, las investigaciones explicativas suelen ser más estructuradas y de hecho implican los propósitos de ellas (descripción, exploración y correlación) además de proporcionar el entendimiento del fenómeno. (Hernández et al, 1997)

En continuidad con lo explicado con anterioridad, la presente investigación empleará el método fenomenológico pues este permite analizar el sujeto de estudio a partir de la realidad que este vive, tanto interna como externamente, pues dicha realidad radica su esencia en el modo como el sujeto vive y percibe el fenómeno. En otras palabras "La fenomenología es el estudio de los fenómenos tal como son experimentados, vividos y percibidos por el hombre. Husserl acuño el término Lebenswelt (mundo de vida, mundo vivido) para expresar la matriz de este "mundo vivido, con su propio significado". (Martínez, 1996, p.167). En este método de investigación, el investigador intenta despojarse de la mayor cantidad de prejuicios y esquemas teóricos para así poder analizar de modo objetivo el sujeto de estudio en torno a la relación con el fenómeno, sin intentar alterar el orden de sus vivencias.

En el proceso de recolección de la información para la investigación cualitativa deben tenerse en cuenta tres aspectos fundamentales como los son: la definición de la estrategia de muestro y el proceso de seleccionar a los participantes, los cuales se orientan en dos principios básicos, la pertenencia y la adecuación, según Sandoval (2002). El primer término hace referencia a identificar aquellos participantes que puedan generar mayores aportes a la investigación y el segundo término se refiere a poder contar con la suficiente recopilación de datos por parte de los participantes para el enriquecimiento de la investigación.

En consecuencia, a lo anterior y teniendo en cuenta los principios básicos de la selección de participantes, la población con la cual se llevará a cabo la presente investigación es con los estudiantes de la Institución Educativa San Francisco de Sales, en donde se tomará como muestra a los estudiantes de grado Noveno y Décimo. Será un muestreo a conveniencia con una totalidad de 50 participantes entre hombres y mujeres.

Dos aspectos fundamentalmente: de un lado, a la elección del lugar, la situación o el evento que más faciliten la labor de registro, sin crear interferencias. Por el otro, a la adopción de una alternativa que le permita al investigador posicionarse socialmente dentro del grupo que busca analizar, mediante una oportuna y bien definida ubicación mental y cultural, a través de la cual obtenga una comprensión clara de la realidad que está estudiando. (Sandoval, 2002, p. 136)

En consecuencia a lo anterior, se escoge a esta población dado a que se encuentran en la mitad de su formación académica en el bachillerato y en los años posteriores iniciaran a ejercer su liderazgo y participación en grados superiores, por lo cual es de importancia promover en ellos ejercicios de participación como sujetos políticos, empleando la gobernanza como herramienta, de igual manera porque debido a la etapa de desarrollo en que se encuentran, los datos recopilados y que puedan aportar serán pertinentes para esta investigación. 
En el presente estudio se emplearan tres de las técnicas de recolección de la información, las cuales son: observación participante, entrevista semiestructurada y círculos de diálogo, la razón por la cual se utilizaran cada una de ellas, lleva un hilo conductor, en primera instancia la observación participante permitirá involucrarse con los sujetos de estudios y analizar aspectos claves como su actitud participativa, interacción con los otros, modo de expresar ideas y posturas, la observación participante realiza su tarea desde el interior de las realidades humanas que desea abordar, es allí donde realiza un contraste con la mirada externalista, las de formas de observación no interactivas (Sandoval, 2002), aparte de ello, la observación participante.

“emplea, para definir el problema de investigación con referencia a la vida cotidiana de las personas, una estrategia flexible de apertura y cierre. Esto quiere decir que puede comenzar con un problema general, para más tarde definir unos escenarios específicos de análisis" (Sandoval,2002, p.140).

Por otra parte, se utilizará la entrevista semiestructurada, la cual permite de ante mano decidir qué tipo de información se requiere y de igual manera poder establecer preguntas formuladas de forma abierta, ya que permitirán una recolección de información más enriquecedora, analítica y matizada que una entrevistas estructurada; en una entrevista semiestructurada la actitud del entrevistador debe ser flexible, cómoda y abierta a las diferentes respuestas que generan las preguntas, permitiéndole saltar de pregunta en pregunta según las respuestas obtenidas.

La entrevista semiestructurada es: entrevista etnográfica. Se puede definir como una "conversación amistosa" entre informante y entrevistador, convirtiéndose este último en un oidor, alguien que escucha con atención, no impone ni interpretaciones ni respuestas, guiando el curso de la entrevista hacia los temas que a él le interesan. Su propósito es realizar un trabajo de campo para comprender la vida social y cultural de diversos grupos, a través de interpretaciones subjetivas para explicar la conducta del grupo. (Díaz, Tourruco, Martínez, Valera, 2013, p. 164)
Una vez realizadas la observación participante y la entrevista individual semiestructurada se procederá a la técnica del grupo focal, ya que tiene su particularidad en su carácter colectivo, el cual:

Contrasta con la singularidad personal de la entrevista en profundidad. Recibe su denominación de focal por lo menos en dos sentidos: el primero se centra en el abordaje a fondo de un número muy reducido de tópicos o problemas; en el segundo, la configuración de los grupos de entrevista se hace a partir de la identificación de alguna particularidad relevante desde el punto de vista de los objetivos de la investigación, lo que lleva a elegir solamente sujetos que tengan dicha característica, por lo general entre seis y ocho. (Sandoval, 2002, p.145-146).

El grupo focal se ajusta a las necesidades de la presente investigación pues permite la interacción colectiva de los participantes, en donde expresaran sus ideas, puntos de vistas, posturas y nociones, en donde se promoverá un ambiente participativo que contribuya a que asuman su rol como sujetos políticos de la mano al conocimiento de la gobernanza como herramienta de participación y construcción ciudadana.

El análisis de la información en la presente investigación se realizará a través de la teoría fundamentada, la cual es definida por Corbin y Strauss (1998) como una "teoría derivada de datos recopilados de manera sistemática y analizados por medio de un proceso de investigación. En este método, la recolección de datos, el análisis y la teoría que surgirá de ellos guardan estrecha relación entre sí" (p.21), es decir, en esta se articulan los datos recopilados, se llevan análisis para posteriormente iniciar a formular teoría a partir del objeto y sujeto de estudio investigado y los resultados encontrados.

\section{Resultados y discusión}

El estudio de la gobernanza y la participación para el desarrollo de sujetos políticos es un tema que aborda holísticamente a la comunidad y la sociedad en general, dado que, es función de los ciudadanos, ser partícipes de las decisiones gubernamentales que 
se toman en su nación, ejerciendo así su rol como sujetos políticos.

De modo que el análisis del uso de la gobernanza para la participación y desarrollo de estudiantes como sujetos políticos en la institución Educativa San Francisco de Sales de la ciudad de Cúcuta, se desarrolló a través de la observación participante, evidenciando en primera instancia, los beneficios que estos proporcionan a la comunidad estudiantil, pues esta permite desarrollar en los jóvenes de temprana edad el interés por la política y la toma de decisiones que afecten su entorno o realidad y la de los demás.

Del mismo modo se empleó una prueba piloto por medio de la cual se buscó evidenciar el concepto que los jóvenes poseen sobre la gobernanza y su incidencia en la participación para el desarrollo como sujetos políticos, por medio de la actividad del círculo de dialogo en el que participaron un grupo aproximado de 15 estudiantes de los grados Noveno y Décimo, seleccionados de forma aleatoria; se les realizó un total de cinco pregunta de manera abierta durante el desarrollo del círculo, como por ejemplo ¿Quién soy yo?, ¿Cómo participo de manera activa en las decisiones sociales? y ¿Qué es para mí la gobernanza? Entre otras que permitían alternar las respuestas estructuradas con las preguntas que iban surgiendo de forma espontánea produciendo respuestas mas críticas y menos mentalizadas, por medio de las cuales ellos podían expresar lo que entendían y consideraban que era la gobernanza y la partición política, pero también como esta incidía en ellos a la hora de participar en las decisiones colectivas, que juegan un papel fundamental tanto en su comunidad educativa como en su círculo social.

Como resultado después de haber realizado una triangulación de la información obtenida desde el marco teórico, los antecedentes y las respuestas dadas por los jóvenes durante la prueba piloto en donde el participante 1 dijo que para él "la participación política es cuando una persona mayor de 18 años puede elegir su gobernante y el alcalde de la ciudad" y el participante 5 contexto que la "gobernanza no es mas que las decisiones que toma el gobierno para el bien del pueblo". Se logró evidenciar que su conocimiento acerca de los conceptos de gobernanza y participación política están un poco fuera de contexto debido que tienen una confusión de lo que es gobernanza y gobernabilidad, para ellos gobernanza es una jerarquización y sobre todo el poder que tiene una sola persona sobre las decisiones de un pueblo, y la participación política la relacionan con aquella intervención que realizan al momento de elegir en las diferentes votaciones que se realizan cada cierto tiempo en el país a los representantes gubernamentales de una comunidad en general. Por ende, no se halla una compresión más allá de la concepción de los conceptos de gobernanza y participación política relacionada a las elecciones de sus gobernantes, más allá de esto, no conciben que los seres humanos son sujetos políticos en su día a día y en su actuar como integrantes de una sociedad, asumiendo posturas, compartiendo su ideología o tomando decisiones en conjunto las cuales ayuden en la obtención del bien común de los implicados en esta.

Es por esto que una institución educativa, la comunidad, la familia, el barrio o la ciudad donde viven los adolescentes se convierten en espacios que inciden en generar acciones o situaciones del diario vivir conllevando a partir de estas, a las posibilidades de participación y el establecimiento de relaciones que posibilitan una postura social.

En efecto a lo anterior, resulta necesaria la implementación de las técnicas de recolección de la información más a fondo, basándose en la entrevista semiestructurada y grupo focal con la totalidad de los jóvenes de los grados Noveno y Décimo.

Añadido a ello, desde el campo disciplinar del trabajo social, fortalece su campo de intervención desde la línea de investigación de acción socioeducativa pues no solo interviene, sino que también profundiza en las concepciones de los estudiantes, su interés en participar como sujetos políticos y sumado a ello el hecho implementar estrategias que conlleven a la formación de seres humanos que se inquieten por ejercer su participación ciudadana, contribuyendo así a la transformación de la sociedad desde el poder del pueblo, generando 
procesos de empoderamiento en estos mismos, para la toma de decisiones a conciencia que contribuyan a la transformación de la realidad social que posee el contexto Colombiano, en miras de encaminar la participación para el alcance de la justicia social, la cual es la justificación ética de la gobernanza y que también es un principio fundamental de la disciplina del trabajo social, pues por medio de esta se contribuye a aumentar el bienestar de los seres humanos.

Como profesión, el trabajo social posee el reto ante la sociedad actual de generar procesos de transformación en los sujetos sociales, el cual no resulta ser sencillo en medio del contexto Colombiano, sin embargo, los jóvenes son la generación promotora del cambio, por ello es fundamental que desde la escuela se pueda contribuir a la formación en participación política de la misma; la gobernanza supone una herramienta fundamental para una construcción de gobierno en un sentido horizontal, aunque actualmente no exista claridad del concepto y su ejercicio en algunos estudiantes, resulta importante dar a conocer el término, como herramienta para la formación y desarrollo de sujetos políticos desde una edad temprana, quienes sean los protagonistas de tomas de decisiones éticas y a conciencia en los próximos años en el marco de la cultura política, competencias ciudadanas, la justicia social y los derechos humanos.

\section{Referencias}

Corbin, J., y Satrauss, A. (1998). Bases de la investigación cualitativa. Técnicas y procedimientos para desarrollar la teoría fundamentada, Universidad de Antioquia, Medellín, Colombia.

Consejo Nacional de Trabajo Social (2013). Código de ética profesional de los trabajadores sociales en Colombia (5a Ed). Bogotá: Editorial Ibáñez. Recuperado de https://trabajosociallibertario. files.wordpress.com/2016/10/codigo-de-etica. pdf
Diaz, L., Torruco, U., Martínez, M., y Valera, M. (2013). La entrevista recurso flexible y dinámico. Investigación en educación média, 2(7), 162167. Recuperado de http://www.scielo.org. $\mathrm{mx} /$ scielo.php?script=sci_arttext\&pid=S2007$50572013000300009 \& \operatorname{lng}=$ es\&tlng=es.

Esteban, P. (21 de mayo de 2017). Los jóvenes y la política. El colombiano. Recuperado de https:// www.elcolombiano.com/opinion/editoriales/ los-jovenes-y-la-politica-YD6577133

Freire, P. (1997). La educación como práctica de la libertad, Madrid, editorial siglo veintiuno de España

Hernández, R., Fernández, C., Baptista, M. (1997). Metodología de la investigación. México D. F. McGRAW-HILL / INTERAMERICANA EDITORES, S.A. DE C.V

Insuasty, A. (2008) La participación en Colombia. Kavilando, ISSN: 2027-2391, Vol 1, No 1 , recuperado de: file://C:/Users/zuly/Downloads/ Dialnet-LaParticipacionEnColombia-4017764. pdf

Le Galès, P. (1998): "Régulation, gouvernance et territoire", en Commaille, J. y Jobert, B. (dirs.): La régulation politique à paraître, Paris, Presses de Sciences Po.

Martínez, M. (1996) Comportamiento Humano. Nuevos Métodos de investigación. Ed. Trillas: México. Segunda parte. métodos estructurales. Cap. 8. El Método fenomenológico Pp.167-188.

Medina, E. (2018) Gobernabilidad y democracia. Bonum Commune. Revista del Instituto Universitario Puebla, Vol. 1, No 0, pp. 57 - 60, recuperado de http://www.bonum.iupyucatan. $\mathrm{mx} / \mathrm{wp}$-content/uploads/2016/06/06_ Art \%C 3\%ADculo_Gobernabilidad-yDemocracia.pdf

Monsalve (2016). Formación política en la escuela. Corregimiento Altavista (Medellín, Colombia): estudio de caso. FORUM Revista departamento de ciencia política, ISSN: 2216-1775 / e-ISSN: 2216-1767 / No 8/9, pp. 75-96, Doi 10.15446/ rf. Recuperado de file:///C:/Users/zuly/ Downloads/67806-383660-1-PB.pdf 
Parada, J. (2010). Democracia y participación en Colombia: un espacio en construcción. Espacio Abierto, 19(4), undefined-undefined. ISSN: 1315-0006. Disponible en: https://www. redalyc.org/articulo.oa?id=122/12216181003

Peña, N. (2017) Los niños y las niñas, ¿sujetos políticos?: construcciones posibles desde la escuela y el aula. Infancias imágenes, Universidad Distrital Francisco José de Caldas, ISSN: 16579089, Vol. 16, Nº 2, Págs.228,241, recuperado de: http://revistas.udistrital.edu.co/ ojs/index.php/infancias.

Retamozo, M. (2009). Lo político y la política: los sujetos políticos, conformación y disputa por el orden social. Perspectivas Teóricas, Revista Mexicana de Ciencias Políticas y sociales ISSN: 2448-492X, Vol 51, No 206, p. 69-91, recuperado de http:/www.revistas.unam.mx/ index.php/rmcpys/article/view/41034/37330

Rodríguez, G, Gil, J., y García, E. (1996). Metodología de la investigación cualitativa. Granada: Aljibe.

Ruano, M. (2002). La gobernanza como forma de acción pública y como concepto analítico, VII Congreso Internacional del CLAD sobre la Reforma del Estado y de la Administración Pública, Lisboa, Portugal, 8-11, Universidad Complutense de Madrid, España.

Sandoval, A. (2003). Investigación cualitativa, programa de especialización en teoría, métodos y técnicas de investigación social, modulo cuatro, ISBN: 958-9329-18-7 Bogotá, Colombia. 